\title{
Optical characterization of chemically doped thin films of poly $(p$-phenylene vinylene)
}

\author{
M. Tzolov a,b,*,1, V.P. Koch ${ }^{\mathrm{b}}$, W. Bruetting ${ }^{\mathrm{a}}$, M. Schwoerer ${ }^{\mathrm{a}}$ \\ ${ }^{a}$ Experimental Physics II, University of Bayreuth, 95440 Bayreuth, Germany \\ b Physics Department E16, Technical University Munich, 85747 Garching, Germany
}

\begin{abstract}
Films of precursor route poly ( $p$-phenylene vinylene) (PPV) have been doped by immersion in $\mathrm{FeCl}_{3}$ solution. The conductivity changes by ca. seven orders of magnitude upon doping and the photoluminescence is quenched. Investigations by photothermal deflection spectroscopy (PDS) have shown the development of broad subbandgap absorption in the spectral range $1.5-2.2 \mathrm{eV}$ and at ca. $0.6 \mathrm{eV}$ upon doping. Similar absorption bands have been detected also for doping with $\mathrm{InCl}_{3}$. The results for undoped samples have shown that the surface of the undoped films is a source of a featureless subgap absorption. The changes in the Raman and infrared spectra with the doping have been investigated. The similarity with the results for films doped with other dopants suggests that the changes are due to transformations of the polymer chains and are independent on the nature of the dopant. It has been shown that the used doping process leads to nonuniform doping of the films. The nonuniformity depends on the film thickness and the concentration of the solution as well.
\end{abstract}

Keywords: Poly( $p$-phenylene vinylene); Doping; Thin films; Optical absorption

\section{I. Introduction}

A variety of models have been developed to explain the operation of the poly ( $p$-phenylene vinylene) (PPV)-based light-emitting diodes (LED). One group is based on the assumption of flat energy band diagram [1]. Another group of models describes the polymer LED as a Schottky diode [2]. Despite the relatively simple structure - transparent conductive oxide/polymer film/metal electrode - many processes in these devices are not completely understood. It has been shown that in the case of precursor route PPV the products from the thermal conversion of the prepolymer react with the ITO substrate [3] and $\mathrm{InCl}_{3}$ is formed. The detailed study [4] led to the conclusion that $\mathrm{InCl}_{3}$ acts as a dopant for the polymer film. Further experiments have shown that the intentional doping with $\mathrm{FeCl}_{3}$ can also influence the device characteristics [5].

\footnotetext{
* Corresponding author. Experimental Physics II, University of Bayreuth, 95440 Bayreuth, Germany. Fax: +49-921-55-2621; e-mail: mtzolov@hotmail.com

${ }^{1}$ On leave from the Central Laboratory for Solar Energy, Sofia, Bulgaria.
}

The immersion of PPV films in a $\mathrm{FeCl}_{3}$ solution is a well known doping method [5,6]. Recent publications about the utilization of conducting polymer anodes [7] and the doping on both sides of the polymer film [8] in LED inspire again the interest in this doping procedure. The polymer doping is interesting also in respect to the applications in other devices like thin film transistors and solar cells. This work concentrates on the doping of thin single layers of precursor route PPV.

\section{Experimental}

The films were prepared via the prepolymer route from a methanol/acetone solution with a doctor blade machine. The temperature conversion of the prepolymer films was performed in inert nitrogen atmosphere for $2 \mathrm{~h}$. The film doping was performed by immersion in $\mathrm{FeCl}_{3}$ solution as described earlier [5] under nitrogen atmosphere. The films for the electrical measurements have not been exposed to ambient air. The thickness of the films was determined by a Dektak 3030 step profiler. Fused quartz substrates were used as substrates for the optical measurements. The infrared measurements were performed on samples deposited 
on c-Si substrates. The surface of these substrates was chemically oxidized before the film deposition. The oxide layer is very thin and does not disturb the infrared measurements. However, it prevents the reaction of the chemical products from the thermal conversion of the prepolymer with the c-Si substrate. The electrical measurements were performed by using interdigitated electrodes formed on the ITO substrate by photolithography. The distance between the electrodes is $500 \mu \mathrm{m}$. Hence the influence of the ITO substrate on the polymer film in the gap between the electrodes is negligible. The film absorption was estimated in the high absorption region from transmission measurements performed with a Lambda 2 spectrophotometer and in the low absorption region from absorption measurements by photothermal deflection spectroscopy (PDS) [9]. The infrared and Raman measurements were performed with a Fourier transform spectrometer Bruker 55. The excitation wavelength for the Raman scattering was $1064 \mathrm{~nm}$. The photoluminescence (PL) spectra were recorded by using pump light at $442 \mathrm{~nm}$ of a GaN LED.

\section{Results and discussion}

With respect to the applications, films with thickness below $100 \mathrm{~nm}$ were mainly studied. The conductivity of 50-nm-thick films after doping in $0.05 \mathrm{wt} \%$ solution for $30 \mathrm{~s}$ grows from $1 \times 10^{-9} \Omega^{-1} \mathrm{~cm}^{-1}$ in the initial state to ca. $10^{-2} \Omega^{-1} \mathrm{~cm}^{-1}$ and further increases slowly with the immersion time. The change of ca. seven orders of magnitude is nearly the same for films converted at $170^{\circ} \mathrm{C}$ and at $300^{\circ} \mathrm{C}$. One has to expect also changes in the optical properties upon doping. The photoluminescence is quenched in all doped samples proportional to the doping level. The changes in the absorption for the above samples are more significant in the spectral range below $2.5 \mathrm{eV}$ (Fig. 1). In this spectral region the data were obtained from PDS measurements. Similar to the previously published data [10] the undoped PPV films show very steep decrease of the absorption coefficient in the vicinity of the band gap followed by a background at low level. The latter values in Fig. 1 are higher than those in Ref. [10] which will be discussed later. Two broad absorption bands grow upon doping in the spectral range $1.5-2.2 \mathrm{eV}$ and below $1.3 \mathrm{eV}$. It has been estimated roughly from the transmission measurements that the low-energy absorption does not increase to lower energies and has its maximum at approximately $0.6 \mathrm{eV}$.

The charges transferred to the polymer chain during the doping process transform the electron configuration within the chains which become quinoid character. The strong electron-phonon coupling leads to the formation of polaron and/or bipolaron states at energies within the band gap. However the species which penetrate into the films during the doping process and which form a charge transfer complex with the PPV chains can also introduce energy

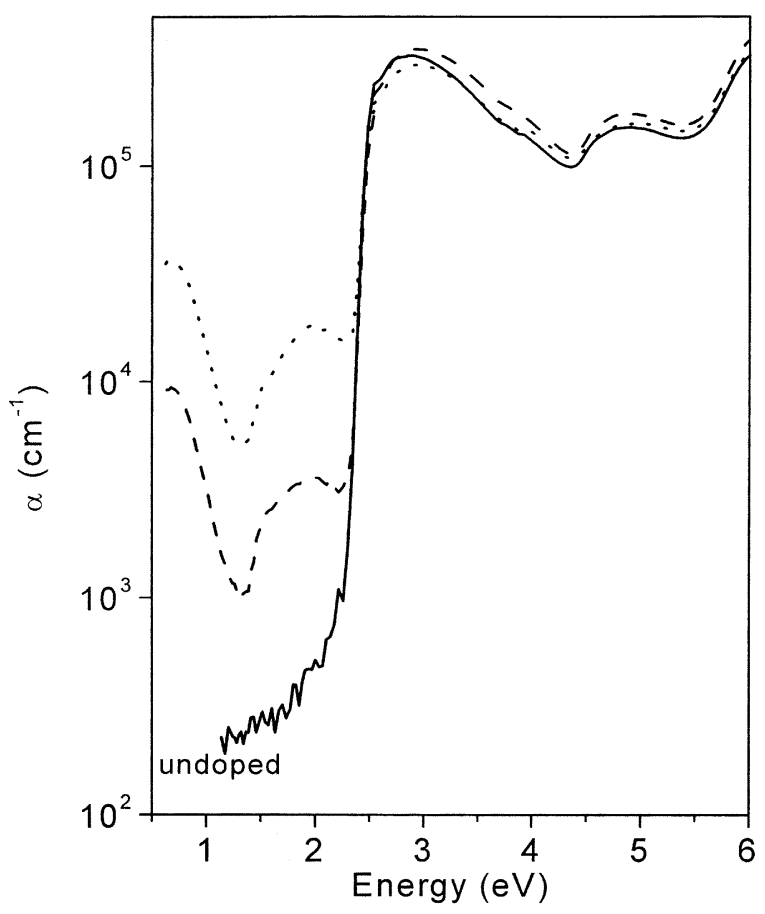

Fig. 1. Absorption coefficient spectra for ca. 50-nm-thick films of PPV converted at $170^{\circ} \mathrm{C}$. The doping was performed in a 0.05 wt. $\% \mathrm{FeCl}_{3}$ solution for $30 \mathrm{~s}$ (dashed line) and $120 \mathrm{~s}$ (dotted line).

levels within the band gap. It is difficult to differentiate these two cases only on the basis of the absorption data. Baitoul et al. [11] have reported the appearance of new bands at 0.8 and $2 \mathrm{eV}$ upon electrochemical oxidative doping by $\mathrm{ClO}_{4}^{-}$. There is a similarity with the data in Fig. 1 which however can not be used as a proof for the origin of the absorption bands.

The effect of the doping can be also seen in the vibrational spectra. Because of the small film thickness (ca. $50 \mathrm{~nm}$ ) the infrared spectrum in Fig. 2 for the undoped sample does not show any well-defined bands. The changes in the film upon doping lead to the appearance of bands at $1138,1273,1319,1419$ and $1508 \mathrm{~cm}^{-1}$. These data agree well with the published data for Na-doped PPV [12]. This similarity suggests that the observed bands are characteristic for the doped polymer chain and are independent of the dopant. The intensity of the doping induced infrared absorption bands increases relative to those for the undoped state because of the change in the dipole moment upon doping.

Similar changes upon doping were observed in the Raman spectra. The vibrational bands for the undoped thin samples lie below the background signal. For that reason the Raman spectrum for a 530-nm-thick film is shown for comparison in Fig. 3, curve A. The Raman spectra for the thin doped films become clearly resolved. The bands at $1172,1329,1415,1548,1585$ and $1626 \mathrm{~cm}^{-1}$ characteristic for the undoped state are present also in the spectra for the doped samples. However the relative intensity of the 


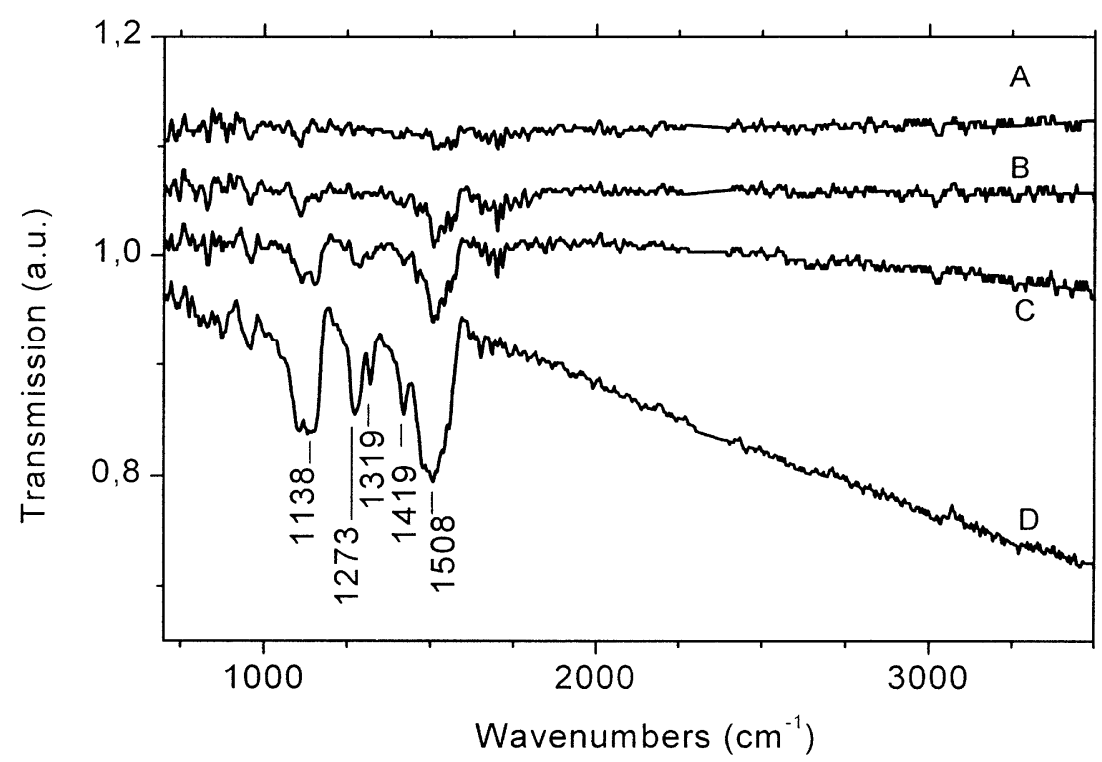

Fig. 2. Transmission spectra for ca. 50-nm-thick samples of PPV converted at $170^{\circ} \mathrm{C}$. The curves are vertically shifted for clarity. Curve A is for an undoped film, curves B and $\mathrm{C}$ are for films doped in $0.05 \mathrm{wt} . \% \mathrm{FeCl}_{3}$ solution for $30 \mathrm{~s}$ and $120 \mathrm{~s}$ respectively, and curve $\mathrm{D}$ is for a film doped in $0.5 \mathrm{wt} . \%$ $\mathrm{FeCl}_{3}$ solution for $10 \mathrm{~s}$. The feature at $1107 \mathrm{~cm}^{-1}$ originates from the c-Si substrate.

band at $1585 \mathrm{~cm}^{-1}$ decreases with the doping level. In addition new bands appear at $1125,1282,1431 \mathrm{~cm}^{-1}$ and a broad feature in the range $1460-1640 \mathrm{~cm}^{-1}$. The increased intensity of the bands characteristic for the undoped states can be attributed to resonance enhancement of the Raman scattering. The absorption band below $1.3 \mathrm{eV}$ in Fig. 1 is in resonance with the laser excitation at 1064 $\mathrm{nm}$ and enhancement of the Raman scattering of the PPV chain modes can take place if this absorption band also originates from the PPV chain. The relative decrease of the band at $1585 \mathrm{~cm}^{-1}$ and the appearance of new bands in the Raman spectrum of the doped samples can be attributed to the transformation of the polymer chains into a quinoid configuration [6,12]. The Raman spectrum in Fig. 3 , curve D is very similar to the one presented by Baitoul et al. [11] for electrochemically doped films. This confirms again the independence of the changes in the vibrational bands on the nature of the dopant.

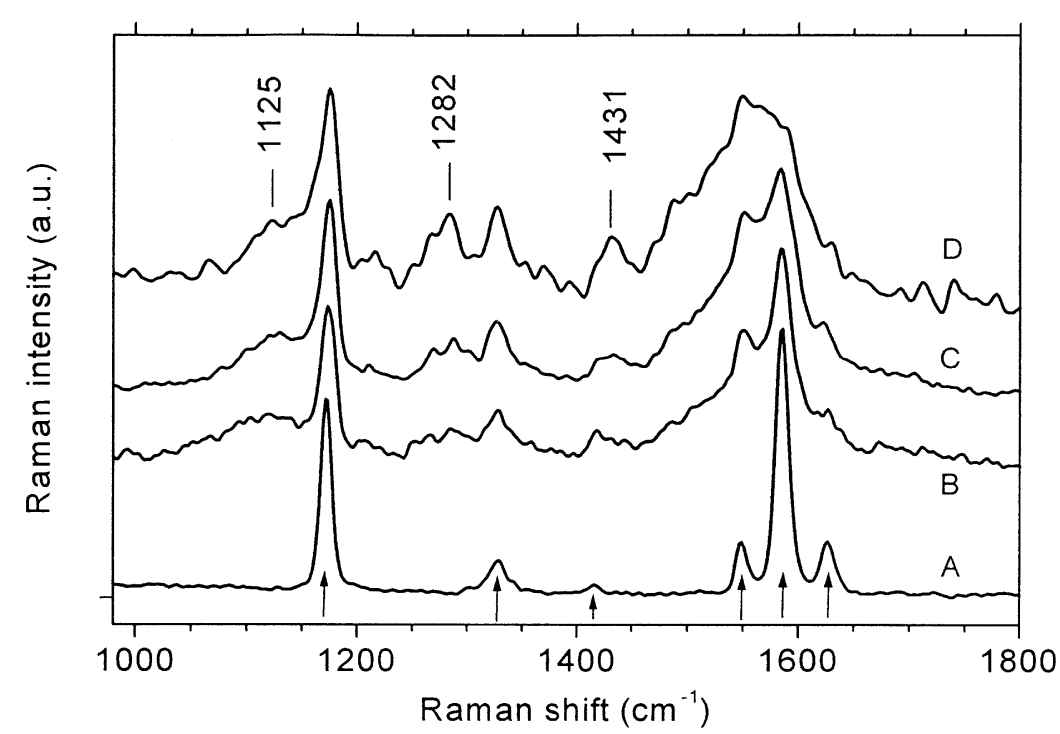

Fig. 3. Raman spectra for samples of PPV converted at $170^{\circ} \mathrm{C}$. The curves are normalized and vertically shifted for clarity. Curves $\mathrm{B}, \mathrm{C}$ and $\mathrm{D}$ are for ca. 50-nm-thick films doped in 0.05 wt.\% solution for $30 \mathrm{~s}, 120 \mathrm{~s}$ and in $0.5 \mathrm{wt} . \%$ solution for $10 \mathrm{~s}$, respectively. Curve A shows the Raman spectrum for 530 -nm-thick undoped sample and the bands typical for the undoped state are designated by arrows. 


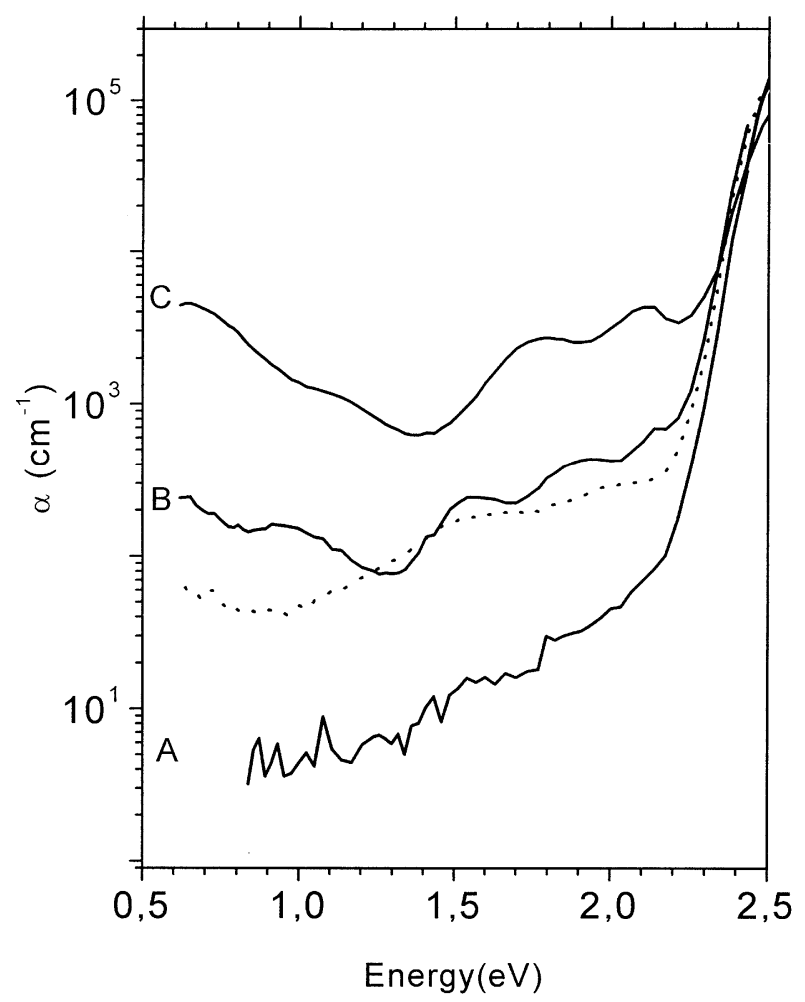

Fig. 4. Absorption coefficient spectra for ca. 500-nm-thick PPV films converted at $170^{\circ} \mathrm{C}$. Curve A is for undoped sample. Curve B is for sample doped in $0.05 \mathrm{wt} . \%$ solution for $30 \mathrm{~s}$ and curve $\mathrm{C}$ for sample doped in $0.5 \mathrm{wt} . \%$ solution for $10 \mathrm{~s}$. The dotted curve is for a 560 -nm-thick sample doped with $\mathrm{InCl}_{3}$ as described in the text.

In order to check the uniformity of the doping, 500nm-thick films have been investigated. The absorption coefficient spectra for the thick samples are shown in Fig. 4 , curves $\mathrm{A}, \mathrm{B}$ and $\mathrm{C}$. The values of the absorption coefficient below the band gap are an order of magnitude lower than those for the thin samples. Because of the higher film thickness the interference fringes are clearly seen for the doped samples. The PDS measurements performed with the pump light illumination through the quartz substrate have shown decreased depth of the interference fringes. Asano and Stutzmann [9] have shown for the case of a-Si:H that such a change in the depth of the interference fringes originates from the existence of a surface layer with higher subgap absorption. If only a film region near the surface is influenced by the doping only this region will show the subgap absorption bands below 2.5 $\mathrm{eV}$ and only here will the photoluminescence be quenched. The nonuniform doping is confirmed also by the photoluminescence measurements using excitation from both sides of the sample. The PL intensity is much higher using an excitation from the quartz substrate, although the spectral shape is the same. Additionally the Raman spectra of the doped thick samples are similar to those of the undoped one because the excitation at $1064 \mathrm{~nm}$ probes the whole film and hence the main contribution to the spectrum originates from the undoped bulk region.
It has been already mentioned that the values of the subgap absorption coefficient are lower for the thicker samples. The calculation of the absorption coefficient was performed using the whole film thickness which leads to lower values in the case of thicker samples if the major contribution comes from the surface region. A similar effect can be observed also in the conductivity.

Fig. 5 shows the thickness variation of the conductivity for two series of different doping levels. The conductivity at higher doping level increases with the film thickness. This dependence can be attributed to the increased influence of the surface recombination at lower film thickness. The photoluminescence is completely quenched in these films which is not the case for the films doped at lower level. The conductivity for the latter films shows the opposite thickness dependence because only a part of the films is doped and the whole film thickness was used in the calculations. Assuming that the thickness of the doped layer is the same $(5 \mathrm{~nm})$ for all films doped at low level one can recalculate the conductivity. The resulting dependence is similar to that for the films doped at higher level.

Most probably the doped and the undoped region are not sharply separated; however, the above results give an idea of the degree of the doping inhomogeneity for the different doping levels.

Attempts were made to dope the PPV films with $\mathrm{InCl}_{3}$ by immersion of converted films in a solution, by mixing of the prepolymer with $\mathrm{InCl}_{3}$ solution and by casting the polymer films over a thin $\mathrm{InCl}_{3}$ film. However either there

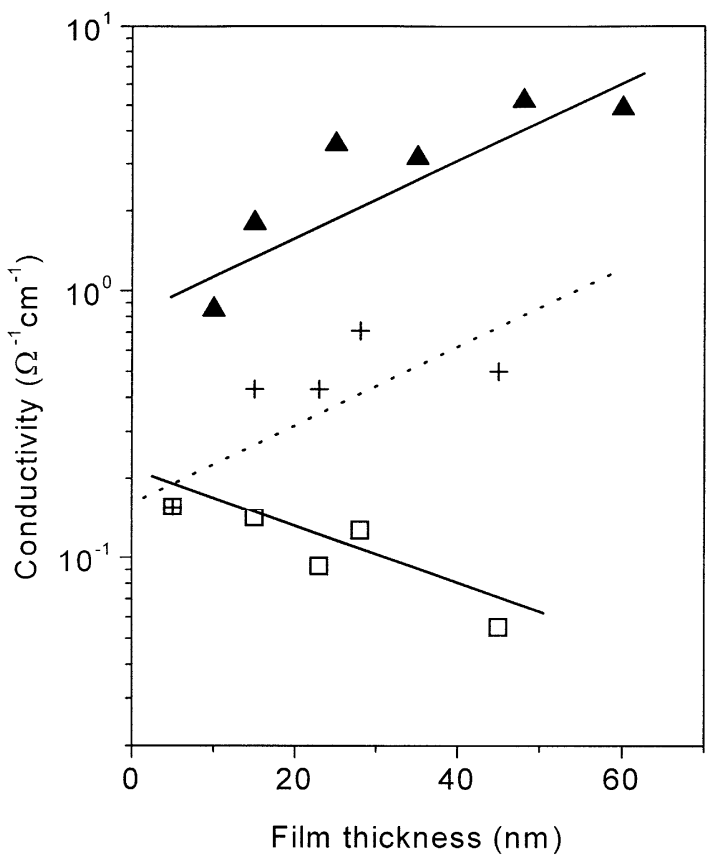

Fig. 5. Conductivity vs. film thickness for PPV samples converted at $170^{\circ} \mathrm{C}$ and doped in $0.5 \mathrm{wt} . \% \mathrm{FeCl}_{3}$ solution for $10 \mathrm{~s}$ (triangles) and in 0.05 wt. $\% \mathrm{FeCl}_{3}$ solution for $120 \mathrm{~s}$ (squares). The crosses represent the latter data calculated with a film thickness of $5 \mathrm{~nm}$. The lines are guides to the eye. 


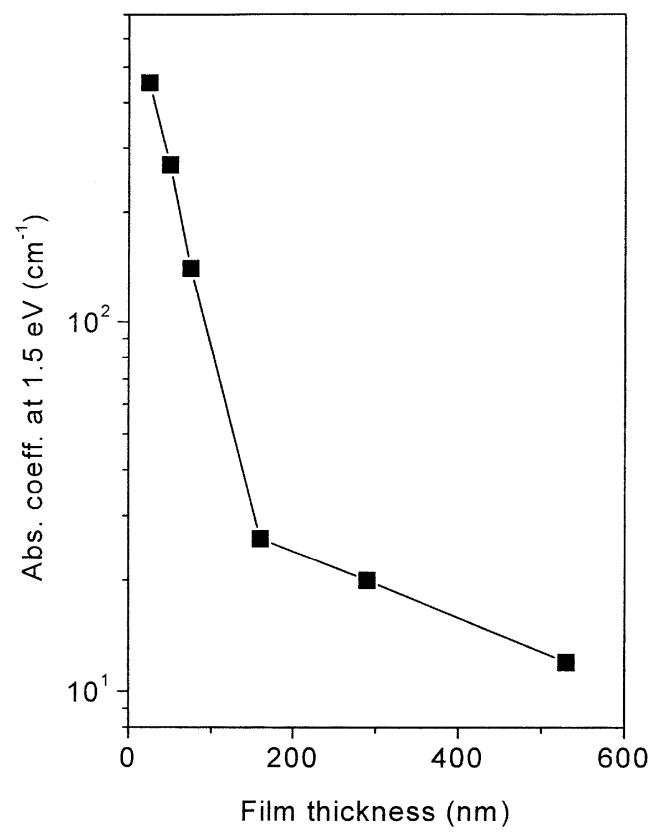

Fig. 6. Subgap absorption vs. film thickness for undoped PPV samples.

was no doping effect or the resulting films were very inhomogeneous. The absorption of polymer films cast on a thin $\mathrm{InCl}_{3}$ films was studied. A typical spectrum for a 560-nm-thick film is shown in Fig. 4, dotted curve. The spectrum shows a broad absorption band between 1 and $2.2 \mathrm{eV}$ and the absorption increases further to lower energies. This result shows that $\mathrm{InCl}_{3}$ can also create states in the band gap. It has to be mentioned that the direct addition of $\mathrm{InCl}_{3}$ to the polymer or prepolymer can differ significantly from the case of thermal conversion of the prepolymer on an ITO substrate [3].

The comparison between the spectra of the undoped samples in Figs. 1 and 4 shows also a thickness dependence of the subgap absorption. The results for undoped PPV films are summarized in Fig. 6. The value of the absorption coefficient at $1.5 \mathrm{eV}$ decreases with the film thickness. The values for the thickest sample are comparable with the results of Saeger et al. [10]. Such a thickness dependence suggests the contribution of surface defect states to the absorption and/or the existence of products of surface oxidation.

\section{Conclusions}

The chemical doping of PPV films in $\mathrm{FeCl}_{3}$ solution stores electrical charge in the polymer chains which leads to transformations of the polymer chains, for example, from benzenoid to quinoid structure. This transformation changes the vibrational properties and the absorption of the films and it seems that they are independent of the nature of the dopant. Despite the simple experimental realization of this doping method many aspects have to be considered in order to obtain accurate results. A thickness nonuniform doping has been found which depends on the film thickness and on the concentration of the solution as well. The understanding of the doping process is important in order to improve it and to apply it properly. Further investigations are in progress.

\section{Acknowledgements}

One of the authors (M.Tz.) would like to acknowledge the financial support of the Alexander von Humboldt Foundation. The authors thank Prof. M. Stutzmann for giving us the opportunity to perform the PDS measurements.

\section{References}

[1] I.D. Parker, J. Appl. Phys. 73 (1994) 1656

[2] S. Karg, M. Meier, W. Riess, J. Appl. Phys. 82 (1997) 1951.

[3] M. Herold, J. Gmeiner, C. Drummer, M. Schwoerer, J. Mater. Sci. 32 (1997) 5709.

[4] W. Bruetting, M. Meier, M. Herold, Chem. Phys. 227 (1998) 243.

[5] E. Werner, J. Gmeiner, M. Herold, W. Bruetting, M. Schwoerer, Opt. Mater. 9 (1998) 109.

[6] S. Lefrant, E. Perrin, J.P. Buisson, Synth. Met. 29 (1989) E91.

[7] S.A. Carter, M. Angelopoulos, S. Karg, P.J. Brock, J.C. Scott, Appl. Phys. Lett. 70 (1997) 2067.

[8] J.M. Bharathan, Y. Yang, J. Appl. Phys. 84 (1998) 3207.

[9] A. Asano, M. Stutzmann, J. Appl. Phys. 70 (1991) 5025.

[10] C.H. Seager, M. Sinclair, D. McBranch, A.J. Heeger, G.L. Baker, Synth. Met. 49/50 (1992) 91.

[11] M. Baitoul, J.P. Buisson, S. Lefrant, B. Dulieu, J. Wery, M. Lapkowski, Synth. Met. 84 (1997) 623.

[12] I. Orion, J.P. Buisson, S. Lefrant, Phys. Rev. B 57 (1998) 7050. 